DOI: 10.12957/demetra.2015.18525

\title{
Relação entre o Ferro Dietético e a Hemoglobina Sérica de Pessoas sob Tratamento Hemodialítico
}

\section{Relationship between Dietary Iron and Hemoglobin Serum People under hemodialysis treatment}

\author{
Nara Conceição Silva Oliveiral \\ Amanda Cibelle de Souza Lima' \\ Josanne Christinne Araújol \\ Fabiane Araújo Sampaio \\ ${ }^{1}$ Faculdade de Ciências e Tecnologia do \\ Maranhão, Departamento de Nutrição. Caxias- \\ MA, Brasil. \\ Correspondência / Correspondence \\ Fabiane Araúio Sampaio \\ E-mail: fabianesampaio21@gmail.com
}

\section{Resumo}

Objetivo: Avaliar a relação entre o ferro dietético e a hemoglobina sérica de pacientes sob tratamento hemodialítico. Metodologia: Estudo transversal, envolvendo 38 homens hemodialíticos, com idade entre 31 e 51 anos. Foram coletados dados de consumo alimentar, por meio do recordatório de 24 horas e analisados pelo software "Nutwin" versão 1.5. As medidas antropométricas foram aferidas com o uso de fita métrica e uma balança digital Filizola ${ }^{\circledR}$ com capacidade máxima de $150 \mathrm{~kg}$, onde a classificação do estado nutricional foi realizada segundo o índice de massa corpórea (IMC). Realizou-se a análise estatística dos dados por meio do programa SPSS v. 15.0, utilizando a correlação de Pearson para verificar associação entre as variáveis. Resultados: Os valores médios do consumo de ferro estavam inferiores às recomendações, com média de 6,22 \pm 7,41 mg/dia. As concentrações médias dos parâmetros bioquímicos hemoglobina, hematócrito e hemácias foram, respectivamente, 9,22 \pm 1,48 g/dl, 28,79 \pm 5,33, 3,15 \pm 0,47 , indicando a presença de anemia. Não houve correlação significativa entre a hemoglobina e o ferro dietético $(p<0,05)$. Conclusão: Diante dos resultados, pode-se pressupor que a anemia dos pacientes sob tratamento hemodialítico não foi resultante da baixa ingestão do ferro, mas decorrente de fatores envolvidos no processo de hemodiálise.

Palavras-chave: Anemia. Deficiência de Ferro. Diálise Renal. Insuficiência Renal. 


\section{Abstract}

Objective: To evaluate the relationship between dietary iron and serum hemoglobin in hemodialysis patients. Method: Crosssectional study involving 38 hemodialysis men, aged between 31 and 51 years. Food consumption data were collected through the 24-hour recall and analyzed by software "Nutwin" version 1.5. Anthropometric measurements were taken using measuring tape and a digital scale Filizola ${ }^{\circledR}$ with a maximum capacity of $150 \mathrm{~kg}$, where the nutritional status was performed according to the body mass index (BMI). There was statistical analysis using the SPSS program v. 15.0, using Pearson's correlation to check the association between variables. Results: The mean iron intake was lower than those recommendations, averaging 6.22 $\pm 7.41 \mathrm{mg} /$ day. The average concentrations of biochemical parameters hemoglobin, hematocrit and red blood cells were, respectively, $9.22 \pm 1.48 \mathrm{~g} / \mathrm{dl}, 28.79 \pm 5.33,3.15 \pm 0.47$, indicating the presence of anemia. There was no significant correlation between hemoglobin and iron in the diet $(\mathrm{P}<0.05)$. Conclusion: Considering the results, it can be assumed that anemia of hemodialysis patients was not the result of low iron intake, but due to factors involved in the hemodialysis process.

Key words: Iron Deficiency. Anemia. Renal Insufficiency. Renal Dialysis.

\section{Introdução}

Os rins são órgãos fundamentais para a manutenção da homeostase do corpo humano, uma vez que a queda progressiva do ritmo de filtração glomerular (RFG) compromete as funções regulatórias, excretórias e endócrinas do organismo. A insuficiência crônica desse órgão é caracterizada por ser uma doença progressiva e debilitante, que causa incapacidade e apresenta alta mortalidade, devido à elevada incidência e prevalência mundial., ${ }^{1,2}$

Pacientes com insuficiência renal crônica necessitam do processo de hemodiálise, tratamento que realiza a filtração sanguínea e elimina resíduos tóxicos, que podem causar prejuízos ao organismo, quando em excesso. No entanto, a hemodiálise traz complicações fisiológicas, psicológicas e nutricionais., ${ }^{2,3}$

Muitos nutrientes são perdidos durante a hemodiálise, incluindo os elementos-traços ou microminerais, dentre eles o ferro, que de acordo com Silva et al., ${ }^{4}$ são minerais necessários para a manutenção da normalidade metabólica e funcionamento adequado das células. Por isso, 
torna-se cautelosa sua suplementação, visto que sua excreção aumentada durante o processo de hemodiálise pode favorecer o desenvolvimento de anemia nesses indivíduos. Além disso, os pacientes hemodialíticos possuem restrições dietéticas que interferem no fornecimento insuficiente do mineral e ainda a utilização de suplementos a base de cálcio ou quelantes de fósforo podem comprometer a absorção do ferro. ${ }^{4-6}$

Outro aspecto que deve ser mencionado é a produção de eritropoetina pelos rins, proteína responsável pela produção de eritrócitos. Em situações de insuficiência dos rins, ocorre a diminuição da síntese de eritropoetina pelo parênquima renal, o que também pode favorecer o desenvolvimento de anemia.,8

A dieta é essencial no tratamento da doença renal crônica, pois a quantidade de proteínas, calorias e outros nutrientes da dieta deve ser corrigida para atender às novas necessidades para melhoria dos sinais clínicos, obterem êxito na terapia, manter ou recuperar o estado nutricional dos pacientes. ${ }^{9,10}$

Com base na escassez de dados que investiguem a influência da hemodiálise no estado nutricional relativo ao ferro e na complexidade do tema, a realização deste estudo foi de suma importância, uma vez que contribuiu para esclarecer os mecanismos envolvidos no desenvolvimento da anemia em pacientes hemodialíticos.

\section{Metodologia}

Trata-se de um estudo transversal realizado com 38 homens sob tratamento hemodialítico, com idade entre 31 e 51 anos, selecionados por meio de entrevistas. Todos os participantes fizeram parte da demanda espontânea do Centro de Diálise de Caxias-Maranhão entre os meses de fevereiro e maio de 2014 e todos assinaram o Termo de Consentimento Livre e Esclarecido. Foram excluídos indivíduos fumantes, alcoólatras e/ou aqueles que apresentaram doenças hepáticas e utilizavam suplementos vitamínico-minerais e outros medicamentos que pudessem interferir no diagnóstico de anemia.

O peso corporal foi determinado por meio de uma balança digital Filizola ${ }^{\circledR}$ com capacidade máxima de $150 \mathrm{~kg}$, graduada em $100 \mathrm{~g}$, estando os participantes descalços e usando roupas leves. A estatura foi obtida com estadiômetro marca Seca®, graduado em centímetros e com barra de madeira vertical e fixa, estando os participantes descalços e sem adereços, com os pés unidos, em posição ereta, olhando para frente. O peso foi medido em quilogramas e a estatura em centímetros, sendo a classificação do estado nutricional dos participantes realizada pelo índice de massa corpórea, segundo a Organização Mundial da Saúde (OMS). ${ }^{11}$ A técnica para coleta de dados do consumo alimentar foi o recordatório de 24 horas, e a quantidade de energia, macronutrientes e o 
ferro foi calculada pelo software "Nutwin", versão 1.5, do Departamento de Informática em Saúde da Universidade Federal de São Paulo. ${ }^{12}$

Os alimentos não encontrados no programa foram incluídos,tomando por base a Tabela Brasileira de Composição de Alimentos. ${ }^{13} \mathrm{O}$ percentual médio de adequação do consumo dos minerais foi calculado com base nas Dietary Reference Intakes.

A anemia foi determinada pela concentração de hemoglobina obtida a partir do método cianometaemoglobina utilizando o Kit Labtest ${ }^{\circledast}$. O espectrofotômetro UV visível (FEMTO modelo $700 \mathrm{~S}$ ) foi utilizado para leitura da hemoglobina, em um comprimento de onda de 540nm. Os dados foram organizados em planilhas do Excel® e exportados para o programa SPSS for Windows ${ }^{\circledR}$ versão 18.0 para análise estatística dos resultados e foram utilizados os testes Kolmogorov-Smirnov para verificar a normalidade dos dados e a correlação de Pearson para verificar associações entre as variáveis, adotando-se um intervalo de confiança de $95 \%$.

O projeto foi encaminhado ao Comitê Centro de Ensino Superior de Caxias-UEMA e aprovado com número do CAAE 26588914.5.0000.5554, conforme prevê a Resolução nº 466/2012, do Conselho Nacional de Saúde (CNS).

\section{Resultados}

Pôde-se verificar que a média de idade dos pacientes encontra-se dentro do ciclo de vida do adulto. Em relação à média do IMC, os pacientes apresentaram-se em risco nutricional de acordo com os novos parâmetros adotados para essa população, conforme os valores médios apresentados na tabela 1 .

Tabela 1. Valores médios e desvios-padrão da idade e dos parâmetros antropométricos, peso, estatura e IMC encontrados no perfil dos pacientes sob tratamento hemodialítico, Caxias-MA, 2014.

\begin{tabular}{cc}
\hline Parâmetros & Pacientes sob tratamento hemodialítico \\
& Média \pm DP \\
\hline Idade $(\operatorname{anos})$ & $41,73 \pm 9,88$ \\
Peso $(\mathrm{kg})$ & $61,28 \pm 10,43$ \\
Estatura $(\mathrm{m})$ & $1,64 \pm 0,08$ \\
IMC $\left(\mathrm{kg} / \mathrm{m}^{2}\right)$ & $22,62 \pm 3,29 *$ \\
\hline
\end{tabular}

IMC= índice de Massa Corpórea; IMC $<23 \mathrm{~kg} / \mathrm{m}^{2}$ apresentam risco nutricional. *Cuppari ${ }^{14}$ 
Os valores médios para energia e macronutrientes encontrados nas dietas consumidas pelos pacientes sob tratamento hemodialítico estão descritos na tabela 2. Verifica-se que houve baixo consumo de energia e lipídios, carboidratos adequados e proteínas acima do recomendado.

Tabela 2. Valores médios e desvios-padrão do consumo alimentar de macronutrientes dos pacientes sob tratamento hemodialítico, Caxias-MA, 2014.

\begin{tabular}{ccc}
\hline Parâmetros & Média $\pm \mathrm{DP}$ & Valores de recomendação \\
\hline Energia $(\mathrm{kcal})$ & $1379,4 \pm 389,65$ & - \\
Proteína $(\mathrm{g} / \mathrm{kg} / \mathrm{dia})$ & $1,32 \pm 0,62$ & $1,2^{*}$ \\
Lipídeo $(\%)$ & $23,8 \pm 7,58$ & $25-35^{\#}$ \\
Carboidrato $(\%)$ & $55,88 \pm 10,56$ & $50-60^{\#}$ \\
\hline
\end{tabular}

${ }^{*}$ Cuppari $^{15}$ Riella e Martins ${ }^{16}$

Os resultados da análise do consumo de ferro e fibra alimentar dos pacientes sob tratamento hemodialítico revelaram baixo consumo desses nutrientes (tabela 3).

Tabela 3. Valores médios e desvios-padrão do consumo alimentar dos micronutrientes, fibra alimentar e ferro dos pacientes sob tratamento hemodialítico, Caxias-MA, 2014.

\begin{tabular}{ccc}
\hline Parâmetros & Média \pm DP & Valores de recomendação \\
\hline Fibra alimentar $(\mathrm{g})$ & $7,89 \pm 7,39$ & 20 a 25 \\
Ferro $(\mathrm{mg})$ & $6,22 \pm 7,41$ & 10 a 18 \\
\hline
\end{tabular}

Cuppari $^{15}$

Os valores médios e desvios-padrão dos parâmetros bioquímicos referentes à determinação da anemia estão disponibilizados na tabela 4. Foram encontrados valores abaixo da recomendação para hematócrito, hemácias e hemoglobina. 
Tabela 4. Valores médios e desvios-padrão dos parâmetros bioquímicos hematócrito, hemácias e hemoglobina dos pacientes sob tratamento hemodialítico, Caxias-MA, 2014.

\begin{tabular}{ccc}
\hline Parâmetros & Média \pm DP & Valores de referência \\
\hline Hematócrito $(\%)$ & $28,79 \pm 5,33$ & $38,0-50,0$ \\
Hemácias (milhões $\left./ \mathrm{mm}^{3}\right)$ & $3,15 \pm 0,47$ & $4,3-5,7$ \\
Hemoglobina $(\mathrm{g} / \mathrm{dL})$ & $9,22 \pm 1,48$ & $13,0-17,5$ \\
\hline
\end{tabular}

Os resultados da análise de correlação entre os parâmetros avaliados encontram-se na tabela 5 . O presente estudo não demonstrou correlação significativa entre as concentrações de hemoglobina e ferro dietético.

Tabela 5. Correlação linear simples entre hemoglobina e ferro dietético, Caxias-MA, 2014.

\begin{tabular}{ccc}
\hline Parâmetros & \multicolumn{2}{c}{ Correlação Linear } \\
\hline Hemoglobina (g/dL) x Ferro dietético $(\mathrm{mg})$ & 0,077 & $0,640^{*}$ \\
\hline
\end{tabular}

*Correlação linear de Pearson $(\mathrm{p}<0,05)$.

\section{Discussão}

Este estudo avaliou o consumo alimentar de ferro e os parâmetros, hematócrito, hemácias e hemoglobina em pacientes sob tratamento hemodialítico, bem como investigou a existência de correlação entre os parâmetros bioquímicos e o ferro dietético. Pôde-se verificar que a idade média encontrada caracteriza a população estudada como adultos. De acordo com estudos realizados por Godinho et al., ${ }^{17}$ a maior parte dos pacientes em hemodiálise no Brasil encontrava-se na faixa dos 40 aos 50 anos.

Apesar do quadro debilitante da patologia, os participantes apresentaram eutrofia, segundo a classificação da OMS para o IMC. Resultados semelhantes foram encontrados por Pinto et al. ${ }^{18}$ e Oliveira et al., ${ }^{19}$ que também identificaram eutrofia em pacientes de doenças renais crônicas em hemodiálise. 
No entanto, novos estudos descrevem que a classificação do estado nutricional a partir do IMC em pacientes com insuficiência renal crônica, submetidos a programas de hemodiálise, apresentam algumas diferenças em relação ao ponto de corte apresentado pela OMS. ${ }^{11}$ Segundo Cuppari, ${ }^{14}$ valores de IMC inferiores a $23 \mathrm{~kg} / \mathrm{m}^{2}$ têm sido indicados como indício de risco nutricional para esses pacientes por estarem associados a morbidade e mortalidade.

Além disso, o IMC não é sensível para constatar depleção proteica e também não aponta o aumento da gordura visceral, reforçando a ideia de que indivíduos com valores de IMC normais podem apresentar riscos de forma diferente. Considerando essa nova classificação, os do desse presente estudo encontram-se, em sua maioria, em risco nutricional.

Quanto ao consumo alimentar dos pacientes, revelou-se heterogeneidade na ingestão de macronutrientes, uma vez que houve baixa ingestão de calorias e lipídios e o consumo de proteínas esteve acima do recomendado. Este fato corrobora os achados de IMC abaixo dos níveis aceitos para esses indivíduos.

Por outro lado, os autores Peters et al. ${ }^{20}$ e Valenzuela et al. ${ }^{21}$ encontraram ingestão deficiente de proteínas em pacientes hemodialíticos, relacionando-a à desnutrição energética e proteica decorrente dessa condição. No entanto, o consumo de carboidratos na alimentação encontrava-se acima da recomendação constatada na literatura em ambos os estudos. Segundo Riella e Martins, ${ }^{22}$ os carboidratos devem suprir cerca de $35 \%$ do total de calorias estimadas para o dia, em virtude da absorção constante de glicose para o paciente em hemodiálise.

É relevante mencionar que a ingestão proteica acima do recomendado também mantém o balanço nitrogenado positivo. Segundo Cabral et al., ${ }^{23}$ a necessidade proteica para pacientes em hemodiálise é elevada, devido à grande perda desse nutriente no processo dialítico, o que não ocorre com a quantidade de gorduras, que deve ser equilibrada por causa da incidência de excesso de peso. Já os valores baixos de calorias encontrados podem ter sido influenciados pela escolha do inquérito alimentar estudo, uma vez que o recordatório de 24 horas parece subestimar a quantidade de energia, por depender da memória do entrevistado e sofrer influência do pesquisador no momento da coleta de informações. Para Dodd, ${ }^{24}$ o método apresenta pouca representatividade do consumo habitual, por restringir a variabilidade do consumo de nutrientes em diferentes dias. Entretanto, apesar das limitações, esse método apresenta como característica a rapidez, a praticidade e o baixo custo.

Quanto ao consumo de ferro, observou-se ingestão deficiente desse nutriente, o que parece ter sido influenciado pela dieta hipocalórica dos participantes, uma vez que o baixo consumo energético reduz a disponibilidade de alimentos fontes de ferro. Segundo Cuppari, ${ }^{14}$ as recomendações de ferro dietético para pacientes hemodialíticos são semelhantes às de indivíduos saudáveis entre $10-18 \mathrm{mg} / \mathrm{dia}$. 
A literatura afirma que a carência de ferro induz o desenvolvimento da anemia. Esta, por sua vez, resulta no aumento da morbidade e mortalidade de pacientes em hemodiálise, reduzindo a qualidade de vida desses indivíduos. Rossert ${ }^{25}$ afirma que a anemia resultante da diminuição do número de néfrons contribui para o agravo do processo de fibrose túbulo-intersticial das nefropatias crônicas, por meio da hipóxia e do estresse oxidativo.

Além disso, a anemia compromete inúmeras funções orgânicas, como transporte de oxigênio aos tecidos, as reações de oxidação e redução, a imunidade humoral e celular, a síntese de ácido desoxirribonucleico (DNA) e a síntese de neurotransmissores. ${ }^{26-28}$

A média de consumo de fibras também esteve abaixo da recomendação, corroborando os resultados encontrados por Chaves et al., ${ }^{29}$ que verificaram que indivíduos sob tratamento hemodialítico tiveram ingestão inadequada de fibras e ferro. Sobre este aspecto, pacientes com insuficiência renal crônica possuem frequente constipação intestinal, decorrente da inatividade física, uso de quelantes de fósforo, suplementos de ferro, restrição de alimentos ricos em potássio e fósforo, os quais também são fontes de fibra. Necessitam, portanto, de reeducação alimentar.

As concentrações séricas de hematócrito, hemácias e hemoglobina avaliados apresentaram-se abaixo dos valores de referência, indicativo de anemia nos pacientes em hemodiálise. Resultados semelhantes foram encontrados por Draczevski \& Teixeira, ${ }^{30}$ que também revelaram anemia em indivíduos submetidos a esse tipo de tratamento. Segundo Paiva et al., ${ }^{31}$ a hemoglobina é o parâmetro universalmente utilizado para diagnosticar a anemia, uma vez que possui quantidade significativa de ferro.

Um ponto importante a ser considerado diz respeito aos resultados da análise realizada para investigar a relação entre o ferro dietético e as concentrações de hemoglobina. Nesse aspecto, a ingestão deficiente de ferro parece não ter influenciado a anemia dos participantes, uma vez que não se verificou a existência de correlações entre os parâmetros supracitados.

Sobre esse resultado, deve-se destacar que o diagnóstico de anemia nos participantes pode ser decorrente de outros fatores inerentes à própria insuficiência renal como, por exemplo, a deficiência da proteína eritropoietina, cuja produção é comprometida em indivíduos com falha renal. Segundo Abensur, ${ }^{32}$ a eritropoietina é produzida nos rins, sendo o principal fator relacionado a alterações na eritropoese (síntese de hemácias). Sua redução bloqueia a liberação de ferro dos macrófagos, o que induz a diminuição do mineral disponível para a síntese da hemoglobina.

Além disso, o processo de hemodiálise induz a espoliação de micronutrientes, dentre eles o ferro, mineral que contribui para a produção de eritropoietina. ${ }^{33}$ Dessa forma, a anemia diagnosticada nos participantes do estudo também pode ter sido causada pela eliminação excessiva de ferro durante o processo de hemodiálise. 
Conforme demonstram os estudos de Martins et al., ${ }^{34}$ os maiores problemas nutricionais nesses indivíduos estão relacionados ao acúmulo de metabólitos entre as sessões dialíticas e à perda de nutrientes durante o procedimento. Segundo Canzianni et al. ${ }^{27}$ a anemia se manifesta nas fases que antecedem o tratamento dialítico, portanto o diagnóstico dessa patologia é fundamental para que haja a redução das complicações. Nesse sentido, a hemoglobina deve ser avaliada em todos os pacientes de DRC, por isso preconiza-se que ela deva ser avaliada pelo menos uma vez ao ano. ${ }^{35}$

Assim, pôde-se pressupor que a anemia ferropriva dos participantes do estudo parece ser decorrente da insuficiência renal e do tratamento hemodialítico, e não resultante da baixa ingestão desse micronutriente na alimentação. Contudo, as limitações deste estudo não permitem um resultado concludente, uma vez que as concentrações de ferro sérico, ferritina e capacidade total de ligação ao ferro não foram avaliados.

Considerando a importância destas análises para resultados mais concludentes, Paiva ${ }^{36}$ afirma que a ferritina é uma medida útil, pois usa sangue periférico e determina forte correlação com o ferro em depósito nos tecidos. O ferro sérico corresponde a um parâmetro bastante utilizado, apresentando alteração na presença de processos infecciosos, evidenciando a diminuição de sua concentração em uma infecção. A capacidade total de ligação do ferro usada para analisar o ferro circulante aumenta na deficiência deste mineral, mas diminui durante a inflamação.

\section{Conclusão}

A partir dos resultados deste estudo, pode-se observar que os pacientes sob tratamento hemodialítico possuem ingestão deficiente de ferro e este, por sua vez, não parece influenciar as baixas concentrações dos parâmetros bioquímicos, hemoglobina, hematócrito e hemácias. Nesse sentido, a anemia presente nos participantes parece ser decorrente de múltiplos fatores, alguns associados à insuficiência renal, com redução na produção de hemácias ou da espoliação de minerais durante o processo de hemodiálise.

Diante do exposto, sugere-se a realização de novos estudos que possam esclarecer a relação entre anemia e hemodiálise, bem como auxiliar no desenvolvimento de estratégias para intervenções tanto na prevenção quanto no tratamento de complicações associadas à IRC.

\section{Agradecimentos}

Os autores gostariam de agradecer à pesquisadora Antonia Samara Melo dos Santos, pela assistência técnica durante a coleta de dados. 


\section{Referências}

1. Bastos MG, Carmo WB, Abrita RR, Almeida EC, Mafra D, Costa DMN, et al. Doença renal crônica: problemas e soluções. J. Bras. Nefrol. 2004; XXVI(4):202-215.

2. Kusumoto L, Marques S, Haas VJ, Rodrigues RAP. Adultos e idosos em hemodialise: avaliação da qualidade de vida relacionada à saúde. Acta Paul. Enferm. 2008; 21:152-9.

3. Abensur H. Deficiência de ferro na doença renal crônica. Rev. Bras. Hematol. Hemoter 2010; 32(Sup. 2):95-98.

4. Silva LF, Santos RMA, Souza IM, Costa JAC, Marchini JS. Terapia nutricional na insuficiência renal crônica. Revista da Sociedade Brasileira de Nutrição 2000; 19/20:105-127.

5. Chaves GA, Graça MD, Gallo VDC. Consumo alimentar e estado nutricional de pacientes com doença renal crônica em tratamento dialítico. Revista Brasileira de Ciências da Saúde 2007; III(14):11-17.

6. Marculino AQ. Avaliação nutricional do paciente submetido à hemodiálise [Especialista]. Criciúma: Universidade do Extremo Sul Catarinense; 2004.

7. Cançado RD, Chiattone CS. Anemia de doença crônica. Rev. Bras. Hematol. Hemoter. 2002; 24(2):127-136.

8. Zago MA, Falcão RP, Pasquini R. Hematologia: fundamentos e prática. São Paulo: Ateneu; 2005.

9. Koehnlein EA, Yamada NA, Giannasi ACB. Avaliação do estudo nutricional de pacientes em hemodiálise. Acta Sci. Health Sci. 2008; 30:65-71

10. National Kidney Fundation. Nutrição e a insuficiência renal crônica. New York: NKF; 2007.

11. World Health Organization. Physical status: use and interpretation of anthropometry. Geneva: WHO; 2000.

12. Anção MS, Cuppari L, Draine AS, Singulem D. Programa de apoio à nutrição Nutwin: versão 1.5. São Paulo. Departamento de Informática em Saúde, SPDM, UNIFESP/EPM; 2002.

13. Universidade Estadual de Campinas. Tabela brasileira de composição de alimentos- TACO. 4 ed. Campinas: NEPA-UNICAMP; 2011.

14. Cuparri L. Guia de medicina ambulatorial e hospitalar da EPM-UNIFESP. Nutrição clínica no adulto. Barueri, SP: Manole; 2014.

15. Cuppari L. Guia de nutrição: nutrição clinica do adulto. 2 ed. Barueri, SP: Manole; 2006.

16. Riella CM, Martins C. Nutrição e o Rim. Rio de Janeiro: Guanabara Koogan; 2001.

17. Godinho TM, Lyra TG, Braga PS, Queiroz RA, Alves JA, Craychete AC. et al. Perfil do paciente que inicia hemodiálise de manutenção em hospital público em Salvador, Bahia. J. Bras. Nefrol. 2006; 28(2):96-103. 
18. Pinto DE, Ullmann LS, Burmeister MM, Antonello ICF, Pizzato A. Associações entre ingestão energética, proteica e de fósforo em pacientes portadores de doença renal crônica em tratamento hemodialítico. J. Bras. Nefrol. 2009; 31(4):269-276.

19. Oliveira GTCD, Andrade EIG, Acurcio FA, Cherchiglia ML, Correia MITD. Avaliação nutricional de pacientes submetidos à hemodiálise em centros de Belo Horizonte. Rev. Assoc. Med. Bras. 2012; 58(2):240-247.

20. Peters BSE, Jorgetti V, Martini LA. Influência do hiperparatireoidismo secundário grave no estado nutricional de pacientes com insuficiência renal crônica. Rev. Nutr. 2006; 19:111-118.

21. Valenzuela RGV, Giffone AG, Cuppari L, Canziani MEF. Estado nutricional de pacientes com insuficiência renal crônica em hemodiálise no Amazonas. Rev. Assoc. Med. Bras. 2003; 49(1):72-78.

22. Riella MC, Martins C. Nutrição e o rim. 2 ed. Rio de Janeiro: Guanabara Koogan; 2001.

23. Cabral PC, Diniz ADS, Arruda IKGD. Avaliação nutricional de pacientes em hemodiálise. Rev. Nutr. 2005; 18(1):29-40.

24. Dodd KW, Guenther PM, Freedman LS, Subar AF, Kipnis V, Midthune D, et al. Statistical methods for estimating usual intake of nutrients and foods: a review of the theory. J. Am. Diet Assoc. 2006; 106(10):1640-50.

25. Rossert JA, McClellan WM, Roger SD, Verbeelen DL, Hörl WH. Contribution of anemia to progression of renal disease: a debate. Nephrol. Dial. Transplant 2002; 17:60-66.

26. Abensur H, Bastos MG, Canziani MEF. Aspectos atuais da anemia na doença renal crônica. J. Bras. Nefrol. 2006; 28:104-107.

27. Canziani MEF, Bastos MG, Bregman R, FILHO RP, Tomiyama C, Draibe SA, et al. Deficiência de ferro e anemia na doença renal crônica. J. Bras. Nefrol. 2006; 28:86-90

28. Pinto GM. Deficiência de ferro: resistência ou suscetibilidade a infecções? Revista Médica de Minas Gerais 2008; 18(3):191-196.

29. Chaves GA, Graça MD, Gallo VDC. Consumo alimentar e estado nutricional de pacientes com doença renal crônica em tratamento dialítico. Revista Brasileira de Ciências da Saúde 2007; 3(14):11-17.

30. Draczevski L, Teixeira ML. Avaliaçao do perfil bioquímico e parâmetros hematologicos em pacientes submetidos à hemodiálise. Revista Saúde e Pesquisa 2011; 4(1):15-22.

31. Paiva AA, Rondó PHC, Guerra-Shinohara EM. Parâmetros para avaliação do estado nutricional de ferro. Rev. Saúde Pública 2000; 34:421-426.

32. Abensur H. Especialidades médicas: nefrologia. Rev. Med. (São Paulo) 2012; 91:49-51.

33. Martins C, Riella MC. Nutrição e hemodialise. In: Riella MC, Martins C, Nutrição e o rim. Rio de Janeiro. Guanabara Koogan; 2001. p. 114-131. 
34. Martins C, Cuppari L, Avesani C, Gusmão MG. Terapia nutricional para pacientes em hemodiálise crônica. Projeto Diretrizes. Associação Médica Brasileira, Conselho Federal de Medicina; 2011.

35. Bregman R. Anemia na doença renal crônica. J. Bras. Nefrol. 2009; 31(1):36-41.

36. Paiva AA, Rondo PHC, Guerra-Shinohara EM. Parâmetros para a avaliação do estado nutricional de ferro. Rev. Saúde Pública 2000; 34(4):421-426.

Recebido: $11 / 9 / 2015$

Revisado: 29/9/2015

Aprovado: 21/10/2015 